\title{
Cosmology and Cosmogony in a Cyclic Universe
}

\author{
Jayant V. Narlikar ${ }^{i}$, Geoffrey Burbidge ${ }^{i i}$, R.G. Vishwakarma ${ }^{i i i}$ \\ Inter-University Centre for Astronomy and Astrophysics, Pune 411007, \\ India $^{i}$ \\ Center for Astrophysics and Space Sciences, University of California, San \\ Diego, CA 92093-0424, USA ${ }^{i i}$ \\ Department of Mathematics, Autonomous University of Zacatecas, \\ Zacatecas, ZAC C.P. 98060, Mexico ${ }^{\text {iii }}$
}

\begin{abstract}
In this paper we discuss the properties of the quasi-steady state cosmological model (QSSC) developed in 1993 in its role as a cyclic model of the universe driven by a negative energy scalar field. We discuss the origin of such a scalar field in the primary creation process first described by F. Hoyle and J. V. Narlikar forty years ago. It is shown that the creation processes which takes place in the nuclei of galaxies are closely linked to the high energy and explosive phenomena, which are commonly observed in galaxies at all redshifts.

The cyclic nature of the universe provides a natural link between the places of origin of the microwave background radiation (arising in hydrogen burning in stars), and the origin of the lightest nuclei $(\mathrm{H}, \mathrm{D}$, $\mathrm{He}^{3}$ and $\mathrm{He}^{4}$ ). It also allows us to relate the large scale cyclic properties of the universe to events taking place in the nuclei of galaxies. Observational evidence shows that ejection of matter and energy from these centers in the form of compact objects, gas and relativistic particles is responsible for the population of quasi-stellar objects (QSOs) and gamma-ray burst sources in the universe.

In the later parts of the paper we briefly discuss the major unsolved problems of this integrated cosmological and cosmogonical scheme. These are the understanding of the origin of the intrinsic redshifts, and the periodicities in the redshift distribution of the QSOs.
\end{abstract}

Keywords : cosmology, cosmogony, high energy phenomena 


\section{Introduction}

\subsection{Cosmological models}

The standard cosmological model accepted by the majority at present is centered about the big bang which involves the creation of matter and energy in an initial explosion. Since we have overwhelming evidence that the universe is expanding, the only alternative to this picture appears to be the classical steady-state cosmology, of Bondi, Gold and Hoyle, (Bondi and Gold, 1948, Hoyle, 1948) or a model in which the universe is cyclic with an oscillation period which can be estimated from observation. In this latter class of model the bounce at a finite minimum of the scale factor is produced by a negative energy scalar field. Long ago Hoyle and Narlikar (1964) emphasized the fact that such a scalar field will produce models which oscillate between finite ranges of scale. In the 1960s theoretical physicists shied away from scalar fields, and more so those involving negative energy. Later Narlikar and Padmanabhan (1985) discussed how the scalar creation field helps resolve the problems of singularity, flatness and horizon in cosmology. It now appears that the popularity of inflation and the so-called new physics of the 1980s have changed the 1960s' mind-set. Thus Steinhardt and Turok (2002) introduced a negative potential energy field and used it to cause a bounce from a non-singular high density state. It is unfortunate that they did not cite the earlier work of Hoyle and Narlikar which had pioneered the concept of non-singular bounce through the agency of a negative energy field, at a time when the physics community was hostile to these ideas. Such a field is required to ensure that matter creation does not violate the conservation of matter and energy.

Following the discovery of the expansion of the universe by Hubble in 1929, practically all of the theoretical models considered were of the Friedmann type, until the proposal by Bondi, Gold and Hoyle in 1948 of the classical steady state model which first invoked the creation of matter. A classical test of this model lay in the fact that, as distinct from all of the big bang models, it predicted that the universe must be accelerating (cf Hoyle and Sandage, 1956). For many years it was claimed that the observations indicated that the universe is decelerating, and that this finding disproved the steady state model. Not until much later was it conceded that it was really not possible to determine the deceleration parameter by the classical 
methods then being used. Gunn and Oke (1975) were the first to highlight the observational uncertainties associated with this test. Of course many other arguments were used against the classical steady state model (for a discussion of the history see Hoyle, Burbidge and Narlikar 2000 Chapters 7 and 8). But starting in 1998 studies of the redshift-apparent magnitude relation for supernovae of Type $1 \mathrm{~A}$ showed that the universe $i s$ apparently accelerating (Riess et al. 1998, Perlmutter et al. 1999). The normal and indeed the proper way to proceed after this result was obtained should have been at least to acknowledge that, despite the difficulties associated with the steady state model, this model had all along been advocating an accelerating universe.

It is worth mentioning that McCrea (1951) was the first to introduce vacuum related stresses with equation of state $p=-\rho$ in the context of the steady state theory. Later Gliner (1970) discussed how vacuum-like state of the medium can serve as original (non singular) state of a Friedmann model.

The introduction of dark energy is typical of the way the standard cosmology has developed; viz, a new assumption is introduced specifically to sustain the model against some new observation. Thus, when the amount of dark matter proved to be too high to sustain the primordial origin of deuterium, the assumption was introduced that most of the dark matter has to be non-baryonic. Further assumptions about this dark matter became necessary, e.g., cold, hot, warm, to sustain the structure formation scenarios. The assumption of inflation was introduced to get rid of the horizon and flatness problems and to do away with an embarrassingly high density of relic magnetic monopoles. As far as the dark energy is concerned, until 1998 the general attitude towards the cosmological constant was typically as summarized by Longair in the Beijing cosmology symposium: "None of the observations to date require the cosmological constant" (Longair 1987). Yet, when the supernovae observations could not be fitted without this constant, it came back with a vengeance as dark energy.

Although the popularity of the cosmological constant and dark energy picked up in the late 1990s, there had been earlier attempts at extending the Friedmann models to include effects of vacuum energy. A review of these models, vis-a-vis observations may be found in the article by Carroll and Press (1992).

We concede that with the assumptions of dark energy, non-baryonic dark matter, inflation etc. an overall self consistent picture has been provided 
within the framework of the standard model. One demonstration of this convergence to self consistency is seen from a comparison of a review of the values of cosmological parameters of the standard model by Bagla, et al. (1996), with the present values. Except for the evidence from high redshift supernovae, in favour of an accelerating universe which came 2-3 years later than the above review, there is an overall consistency of the picture within the last decade or so, including a firmer belief in the flat $(\Omega=1)$ model with narrower error bars.

Nevertheless we also like to emphasize that the inputs required in fundamental physics through these assumptions have so far no experimental checks from laboratory physics. Moreover an epoch dependent scenario providing self-consistency checks, e.g. CMB anisotropies, cluster baryon fraction as a function of redshift does not meet the criterion of 'repeatability of scientific experiment'. We contrast this situation with that in stellar evolution where stars of different masses constitute repeated experimental checks on the theoretical stellar models thus improving their credibility.

Given the speculative nature of our understanding of the universe, a sceptic of the standard model is justified in exploring an alternative avenue wherein the observed features of the universe are explained with fewer speculative assumptions. We review here the progress of such an alternative model.

In this model creation of matter is brought in as a physical phenomenon and a negative kinetic energy scalar field is required to ensure that it does not violate the law of conservation of matter and energy. A simple approach based on Mach's principle leads naturally to such a field within the curved spacetime of general relativity described briefly in $\S 2$. The resulting field equations have the two simplest types of solutions for a homogeneous and isotropic universe: (i) those in which the universe oscillates but there is no creation of matter, and (ii) those in which the universe steadily expands with a constant value of $H_{o}$ being driven by continuous creation of matter. The simplest model including features of both these solutions is the Quasi-Steady State Cosmology (QSSC), first proposed by Hoyle, Burbidge and Narlikar (1993). It has the scale factor in the form:

$$
S(t)=\exp \left(\frac{t}{P}\right)\{1+\eta \cos \theta(t)\}, \theta(t) \approx \frac{2 \pi t}{Q},
$$

where $P$ is the long term 'steady state' time scale of expansion while $Q$ is 
the period of a single oscillation.

Note that it is essential for the universe to have a long term expansion; for a universe that has only oscillations without long term expansion would run into problems like the Olbers paradox. It is also a challenge in such a model to avoid running into 'heat death' through a steady increase of entropy from one cycle to next. These difficulties are avoided if there is creation of new matter at the start of each oscillation as happens in the QSSC, and also, if the universe has a steady long term expansion in addition to the oscillations. New matter in such a case is of low entropy and the event horizon ensures a constant entropy within as the universe expands.

The QSSC has an additional attractive feature if one uses the criterion of the Wheeler and Feynman absorber theory of electromagnetic radiation (Wheeler and Feynman, 1945, 1949). This theory provided a very natural explanation of why in actuality the electromagnetic signals propagate into the future, i.e., via retarded solutions, despite the time-symmetry of the basic equations. By writing the theory in a relativistically invariant actionat-a-distance form, Wheeler and Feynman showed that suitable absorptive properties of the universe can lead to the breaking of time-symmetry. As was discussed by Hogarth (1962) and later by Hoyle and Narlikar (1963, $1969,1971)$ who also extended the argument to quantum electrodynamics, the Wheeler-Feynman theory gives results consistent with observations only if the past absorber is imperfect and the future absorber is perfect. This requirement is not satisfied by a simply cyclic universe or by an ever-expanding big bang universe but is satisfied by the QSSC because of expansion being coupled with cyclicity.

One may question as to why one needs to have the Wheeler-Feynman approach to electrodynamics in preference to field theory. The advantages are many, including (i) a satisfactory explanation of the Dirac formula of radiative reaction, (ii) the unambiguous deduction of why one uses retarded solutions in preference to advanced ones and (iii) a resolution of the ultraviolet divergences in quantum electrodynamics. Rather than go into these aspects in detail we refer the reader to a recent review by Hoyle and Narlikar (1995).

Since cosmology seeks to deal with the large-scale properties of the universe, it inevitably requires a strong connection with fundamental physics. In the big bang cosmology particle physics at very high energy is considered very relevant towards understanding cosmology. In the same spirit we believe 
that the action at a distance approach to fundamental physics brings about an intimate link of microphysics with cosmology. The Wheeler-Feynman approach is an excellent demonstration of such a connection.

\subsection{Cosmogony}

In this paper we shall discuss this cosmological model, but first we want to indicate the importance of the observed behavior of the galaxies (the observed cosmogony) in this approach.

Now that theoretical cosmologists have begun to look with favor on the concepts of scalar negative energy fields, and the creation process, they have taken the position that this subject can only be investigated by working out models based on classical approaches of high energy physics and their effects on the global scale. In all of the discussions of what is called precision cosmology there is no discussion of the remarkable phenomena which have been found in the comparatively nearby universe showing that galaxies themselves can eject what may become, new galaxies. We believe that only when we really understand how individual galaxies and clusters etc. have formed, evolve, and die (if they ever do) shall we really understand the overall cosmology of the universe. As was mentioned earlier, the method currently used in the standard model is to suppose that initial quantum fluctuations were present at an unobservable epoch in the early universe, and then try to mimic the building of galaxies using numerical methods, invoking the dominance of non-baryonic matter and dark energy for which there is no independent evidence.

In one sense we believe that the deficiency of the current standard approach is already obvious. The model is based on only some parts of the observational data. These are: all of the details of the microwave background, the abundances of the light elements, the observed dimming of distant supernovae, and the large scale distribution of the observed galaxies. This has led to the conclusion that most of the mass-energy making up the universe has properties which are completely unknown to physics. This is hardly a rational position, since it depends heavily on the belief that all of the laws of physics known to us today can be extrapolated back to scales and epochs where nothing is really testable; and that there is nothing new to be learned.

In spite of this, a very persuasive case has been made that all of the observational parameters can be fitted together to develop what is now be- 
coming widely accepted as a new standard model, the so-called $\Lambda$ CDM model (Spergel et al., 2003). There have been some publications casting doubt on this model, particularly as far as the reality of dark energy and cold, dark matter are concerned (Meyers et al. 2004; Blanchard et al. 2003). It is usual to dismiss them as controversial and to argue that a few dissenting ideas on the periphery of a generally accepted paradigm are but natural. However, it is unfortunately the case that a large fraction of our understanding of the extragalactic universe is being based on the belief that there was a beginning and an inflationary phase, and that the seeds of galaxies all originate from that very early phase.

We believe that an alternative approach should be considered and tested by observers and theorists alike. In this scheme the major themes are (1) that the universe is cyclic and there was no initial big bang, and (2) all of the observational evidence should be used to test the model. As we shall show, this not only includes the observations which are used in the current standard model, but also the properties and interactions of galaxies and QSOs which are present in the local $(z<0.1)$ universe.

Possibly the most perceptive astronomer in recent history was Viktor Ambartsumian the famous Armenian theorist. Starting in the 1950s and 1960s (Ambartsumian, 1965) he stressed the role of explosions in the universe arguing that the associations of galaxies (groups, clusters, etc.) showed a tendency to expand with far larger kinetic energy than is expected by assuming that the gravitational virial condition holds.

We shall discuss the implications of the cluster dynamics in Section 6 . Here we take up the issue emphasized by Ambartsumian that there apparently exist phenomena in nuclei of galaxies where matter seems to appear with large kinetic energy of motion directed outwards. In Section 6 we will also include other phenomena that share the same property, namely explosive creation of matter and energy. We shall refer to such events as mini-creation events.

Since these phenomena appear on the extragalactic scale and involve quasi-stellar objects, active galaxies, powerful radio sources and clusters and groups of galaxies at all redshifts, we believe they must have an intimate connection with cosmology. Indeed, if one looks at standard cosmology, there too the paradigm centers around the 'big bang' which is itself an explosive creation of matter and energy. In the big bang scenario the origin of all of the phenomena is ultimately attributed to a single origin in the very early 
universe. No connection has been considered by the standard cosmologists between this primordial event and the mini-creation events (MCEs, hereafter) that Ambartsumian talked about. In fact, the QSOs and AGN are commonly ascribed to supermassive black holes as 'prime movers'. In this interpretation the only connection with cosmology is that it must be argued that the central black holes are a result of the processes of galaxy formation in the early universe.

In the QSSC we have been trying to relate such mini-creation events (MCEs) directly to the large scale dynamics of the universe. We show in Sections 2 - 4 that the dynamics of the universe is governed by the frequency and power of the MCEs, and there is a two-way feedback between the two. That is, the universe expands when there is a large MCE activity and contracts when the activity is switched off. Likewise, the MCE activity is large when the density of the universe is relatively large and negligible when the density is relatively small. In short, the universe oscillates between states of finite maximum and minimum densities as do the creation phases in the MCEs.

This was the model proposed by Hoyle, Burbidge and Narlikar (1993) and called the quasi-steady state cosmology (QSSC in brief). The model was motivated partly by Ambartsumian's ideas and partly by the growing number of explosive phenomena that are being discovered in extragalactic astronomy. In the following sections we discuss the cosmological model and then turn to the various phenomena which are beginning to help us understand the basic cosmogony. Then we discuss and look at the phenomena themselves in the framework of this cosmology. Finally, we discuss some of the basic problems that have been uncovered by the new observations for which no theoretical explanation has so far been proposed.

\section{Gravitational Equations With Creation Of Matter}

The mathematical framework for our cosmological model has been discussed by Hoyle, Burbidge and Narlikar (1995; HBN hereafter), and we outline briefly its salient features. To begin with, it is a theory that is derived from an action principle based on Mach's Principle, and assumes that the inertia 
of matter owes its origin to other matter in the universe. This leads to a theoretical framework wider than general relativity as it includes terms relating to inertia and creation of matter. These are explained in the Appendix, and we use the results derived there in the following discussion.

Thus the equations of general relativity are replaced in the theory by

$$
R_{i k}-\frac{1}{2} g_{i k} R+\lambda g_{i k}=8 \pi G\left[T_{i k}-f\left(C_{i} C_{k}-\frac{1}{4} g_{i k} C^{l} C_{l}\right)\right],
$$

with the coupling constant $f$ defined as

$$
f=\frac{2}{3 \tau^{2}}
$$

[We have taken the speed of light $c=1$.] Here $\tau=\hbar / m_{P}$ is the characteristic life time of a Planck particle with mass $m_{P}=\sqrt{3 \hbar / 8 \pi G}$. The gradient of $C$ with respect to spacetime coordinates $x^{i}(i=0,1,2,3)$ is denoted by $C_{i}$. Although the above equation defines $f$ in terms of the fundamental constants it is convenient to keep its identity on the right hand side of Einstein's equations since there we can compare the $C$-field energy tensor directly with the matter tensor. Note that because of positive $f$, the $C$-field has negative kinetic energy. Also, as pointed out in the Appendix, the constant $\lambda$ is negative in this theory.

The question now arises of why astrophysical observation suggests that the creation of matter occurs in some places but not in others. For creation to occur at the points $A_{0}, B_{0}, \ldots$ it is necessary classically that the action should not change (i.e. it should remain stationary) with respect to small changes in the spacetime positions of these points, which can be shown to require

$$
C_{i}\left(A_{0}\right) C^{i}\left(A_{0}\right)=C_{i}\left(B_{0}\right) C^{i}\left(B_{0}\right)=\ldots=m_{P}^{2} .
$$

This is in general not the case: in general the magnitude of $C_{i}(X) C^{i}(X)$ is much less that $m_{P}^{2}$. However, as one approaches closer and closer to the surface of a massive compact body $C_{i}(X) C^{i}(X)$ is increased by a general relativistic time dilatation factor, whereas $m_{P}$ stays fixed.

This suggests that we should look for regions of strong gravitational field such as those near collapsed massive objects. In general relativistic astrophysics such objects are none other than black holes, formed from gravitational collapse. Theorems by Penrose, Hawking and others (see Hawking and 
Ellis 1973) have shown that provided certain positive energy conditions are met, a compact object undergoes gravitational collapse to a spacetime singularity. Such objects become black holes before the singularity is reached. However, in the present case, the negative energy of the $C$-field intervenes in such a way as to violate the above energy conditions. What happens to such a collapsing object containing a $C$-field apart from ordinary matter? We argue that such an object does not become a black hole. Instead, the collapse of the object is halted and the object bounces back, thanks to the effect of the $C$-field. We will refer to such an object as a compact massive object (CMO) or a near-black hole (NBH). In the following section we discuss the problem of gravitational collapse of a dust ball with and without the $C$-field to illustrate this difference.

\section{Gravitational collapse and bounce}

Consider how the classical problem of gravitational collapse is changed under the influence of the negative energy $C$-field. First we describe the classical problem which was first discussed by B. Datt (1938). We write the spacetime metric inside a collapsing dust ball in comoving coordinates $(t, r, \theta, \phi)$ as

$$
\mathrm{d} s^{2}=\mathrm{d} t^{2}-a^{2}(t)\left[\frac{\mathrm{d} r^{2}}{1-\alpha r^{2}}+r^{2}\left(\mathrm{~d} \theta^{2}+\sin ^{2} \theta \mathrm{d} \phi^{2}\right)\right]
$$

where $r, \theta, \phi$ are constant for a typical dust particle and $t$ is its proper time. Let the dust ball be limited by $r \leq r_{b}$.

In the above problem we may describe the onset of collapse at $t=0$ with $a(0)=1$ and $\dot{a}(0)=0$. The starting density $\rho_{0}$ is related to the constant $\alpha$ by

$$
\alpha=\frac{8 \pi G \rho_{0}}{3} .
$$

The field equations (2) without the $C$-field and the cosmological constant then tell us that the equation of collapse is given by

$$
\dot{a}^{2}=\alpha\left(\frac{1-a}{a}\right),
$$

and the spacetime singularity is attained when $a(t) \rightarrow 0$ as $t \rightarrow t_{S}$, where 


$$
t_{S}=\frac{\pi}{2 \sqrt{\alpha}} .
$$

Note that we have ignored the $\lambda$ - term as it turns out to have a negligible effect on objects of size small compared to the characteristic size of the universe.

The collapsing ball enters the event horizon at a time $t=t_{H}$ when

$$
r_{b} a\left(t_{H}\right)=2 G M
$$

where the gravitational mass of the dust ball is given by

$$
M=\frac{4 \pi}{3} r_{b}^{3} \rho_{0}=\frac{\alpha r_{b}^{3}}{2 G} .
$$

This is the stage when the ball becomes a black hole.

When we introduce an ambient $C$-field into this problem, it gets modified as follows. In the homogeneous situation under discussion, $C$ is a function of $t$ only. Let, as before $a(0)=1, \dot{a}(0)=0$ and let $\dot{C}$ at $t=0$, be given by $\beta$. Then it can be easily seen that the equation (7) is modified to

$$
\dot{a}^{2}=\alpha\left(\frac{1-a}{a}\right)-\gamma\left(\frac{1-a}{a^{2}}\right)
$$

where $\gamma=2 \pi G f \beta^{2}>0$. Also the earlier relation (6) is modified to

$$
\alpha=\frac{8 \pi G \rho_{0}}{3}-\gamma
$$

It is immediately clear that in these modified circumstances $a(t)$ cannot reach zero, the spacetime singularity is averted and the ball bounces at a minimum value $a_{\min }>0$, of the function $a(t)$.

Writing $\mu=\gamma / \alpha$, we see that the second zero of $\dot{a}(t)$ occurs at $a_{\min }=\mu$. Thus even for an initially weak $C$-field, we get a bounce at a finite value of $a(t)$.

But what about the development of a black hole? The gravitational mass of the black hole at any epoch $t$ is estimated by its energy content, i.e., by,

$$
\begin{aligned}
M(t) & =\frac{4 \pi}{3} r_{b}^{3} a^{3}(t)\left\{\rho-\frac{3}{4} f \dot{C}^{2}\right\} \\
& =\frac{\alpha r_{b}^{3}}{2 G}\left(1+\mu-\frac{\mu}{a}\right) .
\end{aligned}
$$


Thus the gravitational mass of the dust ball decreases as it contracts and consequently its effective Schwarzschild radius decreases. This happens because of the reservoir of negative energy whose intensity rises faster than that of dust density. Such a result is markedly different from that for a collapsing object with positive energy fields only. From (13) we have the ratio

$$
F \equiv \frac{2 G M(t)}{r_{b} a(t)}=\alpha r_{b}^{2}\left\{\frac{1+\mu}{a}-\frac{\mu}{a^{2}}\right\} .
$$

Hence,

$$
\frac{d F}{d a}=\frac{\alpha r_{b}^{2}}{a^{2}}\left\{\frac{2 \mu}{a}-(1+\mu)\right\} .
$$

We anticipate that $\mu \ll 1$, i.e., the ambient $C$-field energy density is much less than the initial density of the collapsing ball. Thus $F$ increases as $a$ decreases and it reaches its maximum value at $a \cong 2 \mu$. This value is attainable, being larger than $a_{\min }$. Denoting this with $F_{\max }$, we get

$$
F_{\max } \cong \frac{\alpha r_{b}^{2}}{4 \mu} .
$$

In general $\alpha r_{b}^{2} \ll 1$ for most astrophysical objects. For the Sun, $\alpha r_{b}^{2} \cong$ $4 \times 10^{-8}$, while for a white dwarf it is $\sim 4 \times 10^{-6}$. We assume that $\mu$, although small compared to unity, exceeds such values, thus making $F_{\max }<1$. In such circumstances black holes do not form.

We consider scenarios in which the object soon after bounce picks up high outward velocity. From (11) we see that maximum outward velocity is attained at $a=2 \mu$ and it is given by

$$
\dot{a}_{\max }^{2} \approx \frac{\alpha}{4 \mu} .
$$

As $\mu \ll 1$, we expect $\dot{a}_{\max }$ to attain high values. Likewise the $C$-field gradient $(\dot{C}$ in this case) will attain high values in such cases.

Thus, such objects after bouncing at $a_{\min }$ will expand and as $a(t)$ increases the strength of the $C$-field falls while for small $a(t) \dot{a}$ increases rapidly as per equation (11). This expansion therefore resembles an explosion. Further, the high local value of the $C$-field gradient will trigger off creation of Planck 
particles. We will return to this explosive phase in section 7 to illustrate its relevance to high energy phenomena.

It is worth stressing here that even in classical general relativity, the external observer never lives long enough to observe the collapsing object enter the horizon. Thus all claims to have observed black holes in X-ray sources or galactic nuclei really establish the existence of compact massive objects, and as such they are consistent with the NBH concept. A spinning $\mathrm{NBH}$, for example can be approximated by the Kerr solution limited to region outside the horizon (- in an NBH there is no horizon). In cases where $\dot{C}$ has not gone to the level of creation of matter, an NBH will behave very much like a Kerr black hole.

The theory would profit most from a quantum description of the creation process. The difficulty, however, is that Planck particles are defined as those for which the Compton wavelength and the gravitational radius are essentially the same, which means that, unlike other quantum processes, flat spacetime cannot be used in the formulation of the theory. A gravitational disturbance is necessarily involved and the ideal location for triggering creation is that near a CMO. The $C$-field boson far away from a compact object of mass $M$ may not be energetic enough to trigger the creation of a Planck particle. On falling into the strong gravitational field of a sufficiently compact object, however, the boson energy is multiplied by a factor, $(1-2 G M / r)^{-1 / 2}$ for a local Schwarzschild metric.

Bosons then multiply up in a cascade, one makes two, two makes four, ..., as in the discharge of a laser, with particle production multiplying up similarly and with negative pressure effects ultimately blowing the system apart. This is the explosive event that we earlier referred to as a minicreation event (MCE). Unlike the big bang, however, the dynamics of this phenomenon is well defined and non-singular. For a detailed discussion of the role of a NBH as well as the mode of its formation, see Hoyle et al. (2000), (HBN hereafter) p. 244-249.

While still qualitative, we shall show that this view agrees well with the empirical facts of observational astrophysics. For, as mentioned in the previous section, we do see several explosive phenomena in the universe, such as jets from radio sources, gamma ray bursts, X-ray bursters, QSOs and active galactic nuclei, etc. Generally it is assumed that a black hole plays the lead role in such an event by somehow converting a fraction of its huge gravitational energy into large kinetic energy of the 'burst' kind. In actuality, we 
do not see infalling matter that is the signature of a black hole. Rather one sees outgoing matter and radiation, which agrees very well with the explosive picture presented above.

\section{Cosmological Models}

The qualitative picture described above is too difficult and complex to admit an exact solution of the field equations (2). The problem is analogous to that in standard cosmology where a universe with inhomogeneity on the scale of galaxies, clusters, superclusters, etc., as well as containing dark matter and radiation is impossible to describe exactly by a general relativistic solution. In such a case one starts with simplified approximations as in models of Friedmann and Lemaitre and then puts in specific details as perturbation. The two phases of radiation-dominated and matter-dominated universe likewise reflect approximations implying that in the early stages the relativistic particles and photons dominated the expansion of the universe whereas in the later stages it was the non-relativistic matter or dust, that played the major role in the dynamics of the universe.

In the same spirit we approach the above cosmology by a mathematical idealization of a homogeneous and isotropic universe in which there are regularly phased epochs when the MCEs were active and matter creation took place while between two consecutive epochs there was no creation (the MCEs lying dormant). We will refer to these two situations as creative and non-creative modes. In the homogeneous universe assumed here the $C$ field will be a function of cosmic time only. We will be interested in the matter-dominated analogues of the standard models since, as we shall see, the analogue of the radiation-dominated state never arises except locally in each MCE where, however, it remains less intense than the $C$-field. In this approximation, the increase or decrease of the scale factor $S(t)$ of the universe indicates an average smoothed out effect of the MCEs as they are turned on or off. The following discussion is based on the work of Sachs, et al. (1996).

We write the field equations (2) for the Robertson-Walker line element with $S(t)$ as scale factor and $k$ as curvature parameter and for matter in the form of dust, when they reduce to essentially two independent equations : 


$$
\begin{gathered}
2 \frac{\ddot{S}}{S}+\frac{\dot{S}^{2}+k}{S^{2}}=3 \lambda+2 \pi G f \dot{C}^{2} \\
\frac{3\left(\dot{S}^{2}+k\right)}{S^{2}}=3 \lambda+8 \pi G \rho-6 \pi G f \dot{C}^{2},
\end{gathered}
$$

where we have set the speed of light $c=1$ and the density of dust is given by $\rho$. From these equations we get the conservation law in the form of an identity :

$$
\frac{d}{d S}\left\{S^{3}\left(3 \lambda+8 \pi G \rho-6 \pi G f \dot{C}^{2}\right)\right\}=3 S^{2}\left\{3 \lambda+2 \pi G f \dot{C}^{2}\right\} .
$$

This law incorporates "creative" as well as "non-creative" modes. We will discuss both in that order.

\subsection{The creative mode}

This has

$$
T_{; k}^{i k} \neq 0
$$

which, in terms of our simplified model becomes

$$
\frac{d}{d S}\left(S^{3} \rho\right) \neq 0
$$

For the case $k=0$, we get a simple steady-state de Sitter type solution with

$$
\dot{C}=m, \quad S=\exp (t / P),
$$

and from (18) and (19) we get

$$
\rho=f m^{2}, \quad \frac{1}{P^{2}}=\frac{2 \pi G \rho}{3}+\lambda .
$$

Since $\lambda<0$, we expect that

$$
\lambda \approx-\frac{2 \pi G \rho}{3}, \quad \frac{1}{P^{2}} \ll|\lambda|,
$$


but will defer the determination of $P$ to after we have looked at the noncreative solutions. Although Sachs, et al. (1996) have discussed all cases, we will concentrate on the simplest one of flat space $k=0$.

The rate of creation of matter is given by

$$
J=\frac{3 \rho}{P} .
$$

As will be seen in the quasi-steady state case, this rate of creation is an overall average made of a large number of small events. Further, since the creation activity has ups and downs, we expect $J$ to denote some sort of temporal average. This will become clearer after we consider the non-creative mode and then link it to the creative one.

\subsection{The non-creative mode}

In this case $T_{; k}^{i k}=0$ and we get a different set of solutions. The conservation of matter alone gives

$$
\rho \propto \frac{1}{S^{3}}
$$

while for (27) and a constant $\lambda$, (20) leads to

$$
\dot{C} \propto \frac{1}{S^{2}}
$$

Therefore, equation (19) gives

$$
\frac{\dot{S}^{2}+k}{S^{2}}=\lambda+\frac{A}{S^{3}}-\frac{B}{S^{4}}
$$

where $A$ and $B$ are positive constants arising from the constants of proportionality in (27) and (28). We now find that the exact solution of (29) in the case $k=0$, is given by

$$
S=\bar{S}[1+\eta \cos \theta(t)]
$$

where $\eta$ is a parameter and the function $\theta(t)$ is given by

$$
\dot{\theta}^{2}=-\lambda(1+\eta \cos \theta)^{-2}\left\{6+4 \eta \cos \theta+\eta^{2}\left(1+\cos ^{2} \theta\right)\right\} .
$$


Here, $\bar{S}$ is a constant and the parameter $\eta$ satisfies the condition: $|\eta|<1$. Thus the scale factor never becomes zero and the model oscillates between finite scale limits

$$
S_{\min } \equiv \bar{S}(1-\eta) \leq S \leq \bar{S}(1+\eta) \equiv S_{\max }
$$

The density of matter and the $C$-field energy density are given by

$$
\begin{gathered}
\bar{\rho}=-\frac{3 \lambda}{2 \pi G}\left(1+\eta^{2}\right), \\
f \dot{C}^{2}=-\frac{\lambda}{2 \pi G}\left(1-\eta^{2}\right)\left(3+\eta^{2}\right),
\end{gathered}
$$

while the period of oscillation is given by

$$
Q=\frac{1}{\sqrt{-\lambda}} \int_{0}^{2 \pi} \frac{(1+\eta \cos \theta) d \theta}{\left\{6+4 \eta \cos \theta+\eta^{2}\left(1+\cos ^{2} \theta\right)\right\}^{1 / 2}} .
$$

The oscillatory solution can be approximated by a simpler sinusoidal solution with the same period :

$$
S \approx 1+\eta \cos \frac{2 \pi t}{Q}
$$

Thus the function $\theta(t)$ is approximately proportional to $t$.

Notice that there is considerable similarity between the oscillatory solution obtained here and that discussed by Steinhardt and Turok (2002) in the context of a scalar field arising from phase transition. The bounce at finite minimum of scale factor is produced in both cosmologies through a negative energy scalar field. As we pointed out in the introduction, Hoyle and Narlikar (1964) [see also Narlikar (1973)] have emphasized the fact that such a scalar field can produce models which oscillate between finite ranges of scale. In the Hoyle-Narlikar paper cited above $\dot{C} \propto 1 / S^{3}$, as opposed to (28), exactly as assumed by Steinhardt and Turok (2002) 38 years later. This is because instead of the trace-free energy tensor of Equation (2) here, Hoyle and Narlikar had used the standard scalar field tensor given by

$$
-f\left(C_{i} C_{k}-\frac{1}{2} g_{i k} C_{l} C^{l}\right)
$$


Far from being dismissed as physically unrealistic, negative kinetic energy fields like the $C$-field are gaining popularity. Recent works by Rubano and Seudellaro (2004), Sami and Toporensky (2004), Singh, et al. (2003) who refer to the earlier work by Hoyle and Narlikar (1964) have adapted the same ideas to describe phantom matter and the cosmological constant. In these works solutions of vacuum field equations with a cosmological constant are interpreted as a steady state in which matter or entropy is being continuously created. Barrow, et al. (2004) who obtain bouncing models similar to ours refer to the paper by Hoyle and Narlikar (1963) where $C$-field idea was proposed in the context of the steady state theory.

\subsection{The Quasi-Steady State Solution}

The quasi-steady state cosmology is described by a combination of the creative and the non-creative modes. For this the general procedure to be followed is to look for a composite solution of the form

$$
S(t)=\exp \left(\frac{t}{P}\right)\{1+\eta \cos \theta(t)\}
$$

wherein $P \gg Q$. Thus over a period $Q$ as given by (35), the universe is essentially in a non-creative mode. However, at regular instances separated by the period $Q$ it has injection of new matter at such a rate as to preserve an average rate of creation over period $P$ as given by $J$ in (26). It is most likely that these epochs of creation are those of the minimum value of the scale factor during oscillation when the level of the $C$-field background is the highest. There is a sharp drop at a typical minimum but the $S(t)$ is a continuous curve with a zero derivative at $S=S_{\min }$.

Suppose that matter creation takes place at the minimum value of $S=$ $S_{\text {min }}$, and that $N$ particles are created per unit volume with mass $m_{0}$. Then the extra density added at this epoch in the creative mode is

$$
\Delta \rho=m_{0} N .
$$

After one cycle the volume of the space expands by a factor exp $(3 Q / P)$ and to restore the density to its original value we should have

$$
(\rho+\Delta \rho) e^{-3 Q / P}=\rho, \text { i.e., } \quad \Delta \rho / \rho \cong 3 Q / P .
$$


The $C$-field strength likewise takes a jump at creation and declines over the following cycle by the factor $\exp (-4 Q / P)$. Thus the requirement of "steady state" from cycle to cycle tells us that the change in the strength of $\dot{C}^{2}$ must be

$$
\Delta \dot{C}^{2}=\frac{4 Q}{P} \dot{C}^{2} .
$$

The above result is seen to be consistent with (40) when we take note of the conservation law (20). A little manipulation of this equation gives us

$$
\frac{3}{4} \frac{1}{S^{4}} \frac{d}{d S}\left(f \dot{C}^{2} S^{4}\right)=\frac{1}{S^{3}} \frac{d}{d S}\left(\rho S^{3}\right) .
$$

However, the right hand side is the rate of creation of matter per unit volume. Since from (40) and (41) we have

$$
\frac{\Delta \dot{C}^{2}}{\dot{C}^{2}}=\frac{4}{3} \frac{\Delta \rho}{\rho},
$$

and from (23) and (24) we have $\rho=f \dot{C}^{2}$, we see that (42) is deducible from (40) and (41).

To summarize, we find that the composite solution properly reflects the quasi-steady state character of the cosmology in that while each cycle of duration $Q$ is exactly a repeat of the preceding one, over a long time scale the universe expands with the de Sitter expansion factor $\exp (t / P)$. The two time scales $P$ and $Q$ of the model thus turn out to be related to the coupling constants and the parameters $\lambda, f, G, \eta$ of the field equations. Further progress in the theoretical problem can be made after we understand the quantum theory of creation by the $C$-field.

These solutions contain sufficient number of arbitrary constants to assure us that they are generic, once we make the simplification that the universe obeys the Weyl postulate and the cosmological principle. The composite solution can be seen as an illustration of how a non-creative mode can be joined with the creative mode. More possibilities may exist of combining the two within the given framework. We have, however, followed the simplicity argument (also used in the standard big bang cosmology) to limit our present choice to the composite solution described here. HBN have used (38), or its approximation 


$$
S(t)=\exp \left(\frac{t}{P}\right)\left\{1+\eta \cos \frac{2 \pi t}{Q}\right\}
$$

to work out the observable features of the QSSC, which we shall highlight next.

\section{The Astrophysical Picture}

\subsection{Cosmological Parameters}

Coming next to a physical interpretation of these mathematical solutions, we can visualize the above model in terms of the following values of its parameters:

$$
\begin{array}{r}
P=20 Q, Q=5 \times 10^{10} \mathrm{yrs}, \eta=0.811 \\
\lambda=-0.358 \times 10^{-56}(\mathrm{~cm})^{-2} .
\end{array}
$$

To fix ideas, we have taken the maximum redshift $z_{\max }=5$ so that the scale factor at the present epoch $S_{0}$ is determined from the relation $S_{0}=$ $\bar{S}(1-\eta)\left(1+z_{\max }\right)$. This set of parameters has been used in recent papers on the QSSC (Narlikar, et al. 2002, 2003). For this model the ratio of maximum to minimum scale factor in any oscillation is around 9.6.

These parametric values are not uniquely chosen; they are rather indicative of the magnitudes that may describe the real universe. For example, $z_{\max }$ could be as high as 10 without placing any strain on the model. The various observational tests seek to place constraints on these values. Can the above model quantified by the above parameters cope with such tests? If it does we will know that the QSSC provides a realistic and viable alternative to the big bang.

\subsection{The Radiation Background}

As far as the origin and nature of the CMBR is concerned we use a fact that is always ignored by standard cosmologists. If we suppose that most of the ${ }^{4} \mathrm{He}$ found in our own and external galaxies (about $24 \%$ of the hydrogen by mass) was synthesized by hydrogen burning in stars, the energy released 
amounts to about $4.37 \times 10^{-13} \mathrm{erg}^{-3}$. This is almost exactly equal to the energy density of the microwave background radiation with $\mathrm{T}=2.74^{\circ} \mathrm{K}$. For standard cosmologists this has to be dismissed as a coincidence, but for us it is a powerful argument in favor of the hypothesis that the microwave radiation at the level detected is relic starlight from previous oscillations in the QSSC which has been thermalized (Hoyle, et al. 1994). Of course, this coincidence loses its significance in the standard big bang cosmology where the CMBR temperature is epoch-dependent.

It is then natural to suppose that the other light isotopes, namely $\mathrm{D},{ }^{3} \mathrm{He}$, ${ }^{6} \mathrm{Li},{ }^{7} \mathrm{Li},{ }^{9} \mathrm{Be},{ }^{10} \mathrm{~B}$ and ${ }^{11} \mathrm{~B}$ were produced by stellar processes. It has been shown (cf. Burbidge and Hoyle, 1998) that both spallation and stellar flares (for ${ }^{2} \mathrm{D}$ ) on the surfaces of stars can explain the measured abundances. Thus all of the isotopes are ultimately a result of stellar nucleosynthesis (Burbidge et al. 1957; Burbidge and Hoyle 1998).

This option raises a problem, however. If we simply extrapolate our understanding of stellar nucleosynthesis, we will find it hard to explain the relatively low metallicity of stars in our Galaxy. This is still an unsolved problem. We believe but have not yet established that it may be that the initial mass function of the stars where the elements are made is dominated by stars which are only able to eject the outer shells while all of the heavy elements are contained in the cores which simply collapse into black holes. Using theory we can construct a mass function which will lead to the right answer (we think) but it has not yet been done. But of course our handwaving in this area is no better than all of the speculations that are being made in the conventional approach when it comes to the "first" stars.

The theory succeeds in relating the intensity and temperature of CMBR to the stellar burning activity in each cycle, the result emphasizing the causal relationship between the radiation background and nuclear abundances. But, how is the background thermalized? The metallic whisker shaped grains condensed from supernova ejecta have been shown to effectively thermalize the relic starlight (Hoyle et al., 1994, 2000). It has also been demonstrated that inhomogeneities on the observed scale result from the thermalized radiation from clusters, groups of galaxies etc. thermalized at the minimum of the last oscillation (Narlikar et al., 2003). By using a toy model for these sources, it has been shown that the resulting angular power spectrum has a satisfactory fit to the data compiled by Podariu et al (2001) for the band power spectrum of the CMBR temperature inhomogeneities. Extending that work 
further we show, in the following, that the model is also consistent with the first- and third- year observations of the Wilkinson Microwave Anisotropy Probe (WMAP) (Page et al. 2003; Spergel et al. 2006).

Following Narlikar et al (2003) we model the inhomogeneity of the CMBR temperature as a set of small disc-shaped spots, randomly distributed on a unit sphere. The spots may be either 'top hat' type or 'Gaussian' type. In the former case they have sharp boundaries whereas in the latter case they taper outwards. We assume the former for clusters, and the latter for the galaxies, or groups of galaxies, and also for the curvature effect. This is because the clusters will tend to have rather sharp boundaries whereas in the other cases such sharp limits do not exist. The resultant inhomogeneity of the CMBR thus arises from a superposition of random spots of three characteristic sizes corresponding to the three effects - the curvature effects at the last minimum of the the scale factor, clusters, and groups of galaxies. This is given by a 7 - parameter model of the angular power spectrum (for more details, see Narlikar et al, 2003):

$$
\begin{aligned}
\mathcal{C}_{l}= & A_{1} l(l+1) e^{-l^{2} \alpha_{1}^{2}} \\
& +A_{2} \frac{l^{\gamma-2}}{l+1}\left[\cos \alpha_{2} P_{l}\left(\cos \alpha_{2}\right)-P_{l-1}\left(\cos \alpha_{2}\right)\right]^{2} \\
& +A_{3} l(l+1) e^{-l^{2} \alpha_{3}^{2}},
\end{aligned}
$$

where the parameters $A_{1}, A_{2}, A_{3}$ depend on the number density as well as the typical temperature fluctuation of each kind of spot, the parameters $\alpha_{1}, \alpha_{2}$, $\alpha_{3}$ correspond to the multipole value $l_{p}$ at which the $\mathcal{C}_{l}$ from each component peaks, and the parameter $\gamma$ refers to the correlation of the hot spots due to clusters. These parameters are determined by fitting the model to the observations by following the method we have used in (Narlikar, et al, 2003). We find that the observations favour a constant in place of the first gaussian profile in equation (46), resulting in a 6-parameter model with $A_{1}, A_{2}, A_{3}$, $\alpha_{2}, \alpha_{3}$ and $\gamma$ as the remaining free parameters. We should mention that the first gaussian profile of equation (46) had been conjectured by Narlikar, et al (2003) to be related to signature of spacetime curvature at the last minimum scale of oscillation. This conjecture was analogous to the particle horizon in the standard cosmology. In the QSSC, there is no particle horizon and the current observations suggest that the curvature effect on CMBR inhomogeneity is negligible. 
For the actual fitting, we consider the WMAP-three year data release (Spergel, et al, 2006). The data for the mean value of TT power spectrum have been binned into 39 bins in multipole space. We find that the earlier fit (Narlikar, et al, 2003) of the model is worsened when we consider the new data, giving $\chi^{2}=129.6$ at 33 degrees of freedom. However, we should note that while the new data set (WMAP-three year) has generally increased its accuracy, compared with the WMAP-one year observations, for $l \leq 700$, the observations for higher $l$ do not seem to agree. This is clear from Figure 1 where we have shown these two observations simultaneously. If we exclude the last three points from the fit, we can have a satisfactory fit giving $\chi^{2}=$ 83.6 for the best-fitting parameters $A_{1}=890.439 \pm 26.270, A_{2}=2402543.93 \pm$ $3110688.86, A_{3}=0.123 \pm 0.033, \alpha_{2}=0.010 \pm 0.0001, \alpha_{3}=0.004 \pm 0.000004$ and $\gamma=3.645 \pm 0.206$, We shall see in the following that the standard cosmology also supplies a similar fit to the data. It should be noted that the above mentioned parameters in the QSSC can be related to the physical dimensions of the sources of inhomogeneities along the lines of Narlikar et al (2003) and are within the broad range of values expected from the physics of the processes.

For comparison, we fitted the same binned data, to the anisotropy spectrum prediction of a grid of open-CDM and $\Lambda$-CDM models within the standard big bang cosmology. We varied the matter density, $\Omega_{\mathrm{m}}=0.1$ to 1 in steps of 0.1 ; the baryon density, $\Omega_{\mathrm{b}} h^{2}$ from 0.005 to 0.03 in steps of 0.004 where $h$ is the Hubble constant in units of $100 \mathrm{~km} \mathrm{~s}^{-1} \mathrm{Mpc}^{-1}$; and the age of the universe, $t_{0}$ from 10 Gyr to 20 Gyr in steps of 2 Gyr. For each value of $\Omega_{\mathrm{m}}$ we considered an open model and one flat where a compensating $\Omega_{\Lambda}$ was added. For the same binned data set, we find that the minimum value of $\chi^{2}$ is obtained for the flat model $\left(\Omega_{\mathrm{m}}=0.2=1-\Omega_{\Lambda}, \Omega_{\mathrm{b}} h^{2}=0.021, t_{0}=14\right.$ Gyr and $h=0.75)$ with $\chi^{2}=95.9$ for the full data and $\chi^{2}=92.7$ from the first 36 points. Though the fit can be improved marginally by fine tunning the parameters further. However, it should be noted that the error bars (we have used) provided by the WMAP team provide only a rough estimate of the errors, not the exact error bars. For a proper assignment of errors, it is suggested to use the complete Fisher matrix. However, one should note that some components that go into making the Fisher matrix, depend on the particular models. This makes the errors model dependent which prohibits an independent assessment of the viability of the model. Hence until the modelindependent errors are available from the observations, we are satisfied by 
our procedures and qualities of fit for both theories.

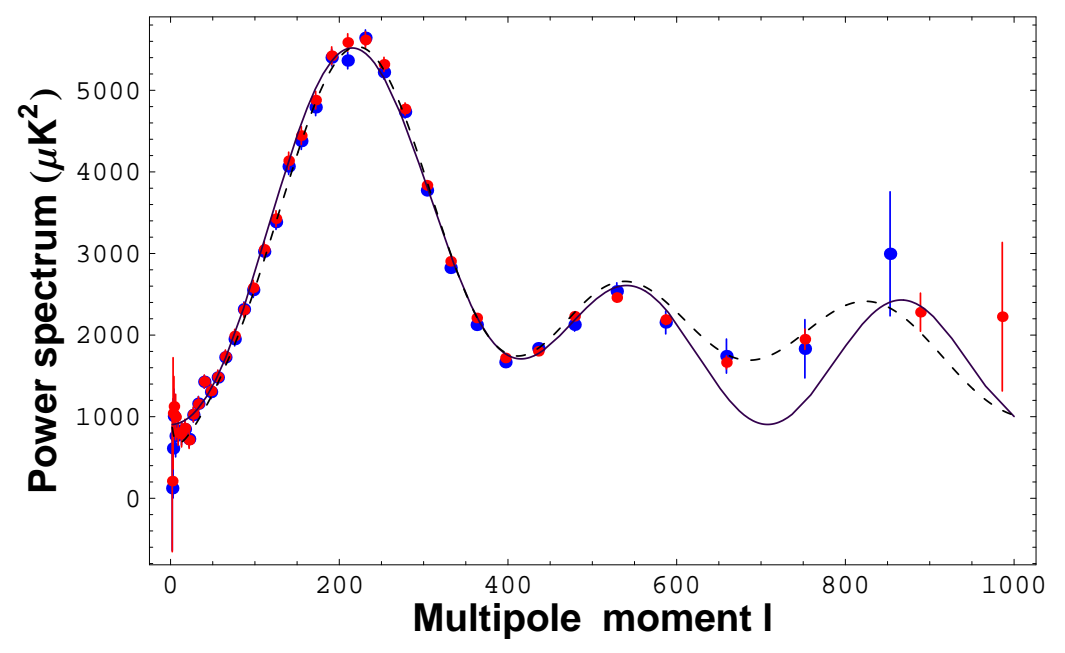

Figure 1: We plot the best-fitting angular power spectrum curves to the WMAPthree year data (shown in red colour) averaged into 39 bins. The continuous curve corresponds to the QSSC with 6 parameters and the dashed one to the big bang model with $\Omega_{\mathrm{m}}=0.2, \Omega_{\Lambda}=0.8$. We notice that the highest parts of contribution to $\chi^{2}$ is from the last three points and the first 4 points of the data, on which the observations have not settled yet, as is clear from the comparision of these data with the WMAP-one year data (shown in blue colour). The rest of the points have reasonable fits with the theoretical curves.

Figure 1 shows the best-fitting angular power spectrum curve obtained for QSSC by using the six parameter model. For comparison, we have also drawn the best-fitting big bang model.

We mention in passing that recent work (Wickramasinghe 2005) indicates that small traces of polarization would be expected in the CMBR wherever it passes through optically thin clouds of iron whiskers. These whiskers being partially aligned along the intracluster magnetic fields will yield a weak signal of polarization on the scale of clusters or smaller ojects. 
It should be noted that the small scale anisotropies do not constitute as crucial a test for our model as they do for standard cosmology. Our general belief is that the universe is inhomogeneous on the scales of galaxycluster-supercluster and the QSSC model cannot make detailed claims of how these would result in the anisotropy of CMBR. In this respect, the standard model subject to all its assumptions (dark matter, inflation, dark energy, etc.) makes much more focussed predictions of CMBR anisotropy.

It is worth commenting on another issue of an astrophysical nature. The typical QSSC cycle has a lifetime long enough for most stars of masses exceeding $\sim 0.5-0.7 M_{\odot}$ to have burnt out. Thus stars from previous cycles will be mostly extinct as radiators of energy. Their masses will continue, however, to exert a gravitational influence on visible matter. The so-called dark matter seen in the outer reaches of galaxies and within clusters may very well be made up, at least in part, of these stellar remnants.

To what extent does this interpretation tally with observations? Clearly, in the big bang cosmology the time scales are not long enough to allow such an interpretation. Nor does that cosmology permit dark matter to be baryonic to such an extent. The constraints on baryonic dark matter in standard cosmology come from (i) the origin and abundance of deuterium and (ii) considerations of large scale structure. The latter constraint further requires the nonbaryonic matter to be cold. In the QSSC, as has been shown before, these constraints are not relevant. For other observational issues completely handled by the QSSC, see Hoyle et al. (2000).

The QSSC envisages stars from previous cycles to have burnt out and remained in and around their parent galaxies as dark matter. These may be very faint white dwarfs, neutron stars and even more massive remnants of supernovae, like near black holes. Their masses may be in the neighbourhood of $M_{\odot}$, or more, i.e., much larger than planetary or brown dwarf masses. Thus one form of baryonic dark matter could be in such remnants. In this connection results from surveys like MACHO or OGLE would provide possible constraints on this hypothesis. We should mention here that unlike the standard cosmology, the QSSC does not have limits on the density of baryonic matter from considerations of deuterium production or formation of large scale structure. 


\section{Explosive Cosmogony}

\subsection{Groups and clusters of galaxies}

We have already stated that it was Ambartsumian (1965) who first pointed out that the simplest interpretation of many physical systems of galaxies ranging from very small groups to large clusters is that they are expanding and coming apart. Since most of the observations are of systems at comparatively small redshifts it is clear that this takes place at the current epoch, and while we do not have direct evidence of the situation at large redshifts, it is most likely a general phenomenon.

Why has this effect been so widely ignored? The answer to this is clearly related to the beliefs of earlier generations of cosmologists. From an historical point of view, the first physical clusters were identified in the 1920s, and it was Zwicky, and later others who supposed that they must be stable systems. By measuring individual redshifts of a number of the galaxies in such a cluster it is possible to get a measurement of the line-of-sight random motions. For stability the virial condition $2 E_{K}+\Omega=O$ needs to be satisfied where $E_{K}$ and $\Omega$ are the average values of the kinetic energy and potential energy of the cluster members. Extensive spectroscopic studies from the 1950s onward showed that nearly always the kinetic energy of the visible matter far exceeds the potential energy apparent from the visible parts of the galaxies. Many clusters have structures which suggest they are stable and relaxed. Thus it was deduced that in these clusters there must be enough dark matter present to stabilize them. This was, originally, one of the first pieces of evidence for the existence of dark matter.

The other argument was concerned with the ages of the galaxies. Until fairly recently it has been argued that all galaxies have stellar populations which include stars which are very old, with ages on the order of $H_{o}^{-1}$, i.e. that they are all as old as the classic big bang universe. However we now know that young galaxies with ages $\ll H_{o}^{-1}$ do exist. But the major point made by Ambartsumian was, and is, that there are large numbers of clusters of galaxies, and many small groups, which are physically connected but clearly from their forms and their relative velocities, appear to be unstable.

In this situation the use of the virial theorem is totally inappropriate. It is worthwhile pointing out that if the virial theorem holds the random motions of the galaxies should follow a steady state distribution such as 


$$
F(\mathbf{v}) \propto \exp \left[-\frac{\mathbf{v}^{2}}{2 \sigma^{2}}\right] .
$$

So far there is no observational demonstration that this is indeed the case. The conclusion drawn from $2 E_{K}+\Omega>O$ as based on visible components only should rather be that the clusters are manifestly not in dynamical equilibrium.

Unfortunately, over the last thirty years the virial approach has been wedded to the idea that all galaxies are old, and it is this mis-reading of the data that led to the view that most galaxies were formed in the early universe and cannot be forming now. For example, in 1974 Ostriker, Peebles and Yahil (1974) argued in a very influential paper that the masses of physical systems of galaxies increase linearly with their sizes. As one of us pointed out at the time (Burbidge, 1975) this result was obtained completely by assuming that at every scale, for binary galaxies, very small groups, larger groups, and rich clusters, the virial condition of stability holds. Thus it was argued that more and more dark matter is present as the systems get bigger.

Modern evidence concerning the masses of clusters has been obtained from x-ray studies, the Sunyaev-Zeldovich effect, and gravitational lensing (cf. Fabian 1994; Carlstrom et al. 2002; Fort and Mellier 1994 and many other papers). All of these studies of rich clusters of galaxies show that large amounts of matter in the form of hot gas and/or dark matter must be present. However, evidence of enough matter to bind small or irregular clusters has not been found in general, and these are the types of configurations which Ambartsumian was originally considering. A system such as the Hercules Cluster is in this category. Also the very compact groups of galaxies (cf. Hickson 1997) have been a subject of debate for many years since a significant fraction of them $(\sim 40 \%)$ contain one galaxy with a redshift very different from the others. Many statistical studies of these have been made, the orthodox view being that such galaxies must be "interlopers"; foreground or background galaxies. Otherwise they either have anomalous redshifts, or are exploding away from the other galaxies.

We also have the problem of interacting galaxies, briefly referred to earlier in Section 1. In modern times it has been generally supposed that when two galaxies are clearly in interaction they must be coming together (merging) and never coming apart. There are valid ways of deciding whether or not mergers are, or have occurred. The clearest way to show that they are 
coming together is to look for tidal tails (Toomre and Toomre 1972), or, if they are very closely interwoven, to look for two centers, or two counter rotating systems. For some objects this evidence does exist, and mergers are well established. But to assume that merging is occurring in all cases is unreasonable: there may well be systems where we are seeing the ejection of one galaxy from another as Ambartsumian proposed. Thus when the virial condition is not satisfied, and the systems are highly irregular and appear to be coming apart, then perhaps they are coming apart, and never have been separate. Here we are clearly departing from the standard point of view.

If one assumes that clusters may not be bound, their overall astrophysics changes from that of bound 'steady' clusters. Issues like the nature of intracluster medium, the role of the halo, generation of x-rays will require a new approach in the case where clusters are expanding. Further, the ejection of new matter provides additional inputs to the dynamics of the system. For example, the energy of ejection will play a role in heating the intracluster

gas. This important investigation still needs to be carried out. However, a preliminary discussion may be found in Hoyle, et al. (2000), Chapter 20.

\subsection{Explosions in individual galaxies}

By the early 1960s it had become clear that very large energy outbursts are taking place in the nuclei of galaxies.

The first evidence came from the discovery of powerful radio sources and the realization that the nuclei of the galaxies which they were identified with, had given rise to at least $10^{59}-10^{61}$ ergs largely in the form of relativistic (Gev) particles and magnetic flux which had been ejected to distances of $\geq$ $100 \mathrm{kpc}$ from the region of production.

A second line of evidence comes from the classical Seyfert galaxies which have very bright star-like nuclei which show very blue continua, and highly excited gas which has random motions $\gtrsim 3000 \mathrm{Km} \mathrm{sec}^{-1}$, and must be escaping from the nucleus. We know that the gas is being ejected because we see it through absorption in optical and X-ray spectra of Seyfert nuclei, and the wavelengths of the absorption lines are shifted to the blue of the main emission. The speeds observed are very large compared with the escape velocity. Early data were described by Burbidge et al. (1963).

In the decades since then it has been shown that many active nuclei are giving rise to x-rays, and to relativistic jets, detected in the most detail as 
high frequency radio waves. A very large fraction of all of the energy which is detected in the compact sources is non-thermal in origin, and is likely to be incoherent synchrotron radiation or Compton radiation.

Early in the discussion of the origin of these very large energies it was concluded that the only possible energy sources are gravitational energy associated with the collapse of a large mass, and the ejection of a small fraction of the energy, or we are indeed seeing mass and energy being created in the nuclei (cf. Hoyle, Fowler, Burbidge and Burbidge 1964).

Of course the most conservative explanation is that the energy arises from matter falling into massive black holes with an efficiency of conversion of gravitational energy to whatever is seen, of order $10 \%$. This is the argument that has been generally advanced and widely accepted (cf. Rees 1984).

Why do we believe that this is not the correct explanation? After all, there is good evidence that many nearby galaxies (most of which are not active) contain collapsed supermassive objects in their centers with masses in the range $10^{6}-10^{8} \mathrm{M}_{\odot}$.

The major difficulty is associated with the efficiency with which gravitational energy can be converted into very fast moving gas and relativistic particles, a problem that has haunted us for more than forty years (Burbidge and Burbidge 1965). In our view the efficiency factor is not $10 \%$ but close to $0.1 \%-1 \%$. The reasons why the efficiency factor is very small are the following. If the energy could be converted directly the efficiency might be as high as $\sim 8 \%$, or even higher from a Kerr rotating black hole. But this energy will appear outside the Schwarzschild radius as the classical equivalent of gravitons. This energy has to be used to heat an accretion disk or generate a corona in a classical AGN, or generate very high energy particles which can propagate outward in a radio source, then heat gas which gives rise to shock waves, which accelerate particles, which in turn radiate by the synchrotron process. Thermodynamics tells us that the efficiency at each of these stages is $\lesssim 10 \%$. If there are 3 to 4 stages the overall efficiency is $\sim 10^{-3}-10^{-4}$. This is borne out by the measured efficiency by which relativistic beams are generated in particle accelerators on earth, and by the efficiency associated with the activity in the center of M87. (cf. Churasov et al. 2002).

If these arguments are not accepted, and gravitational energy is still claimed to be the only reasonable source, another problem appears.

For the most luminous sources, powerful radio sources and distant QSOs the masses involved must be much greater than the typical values used by 
the black hole-accretion disk theorists. If one uses the formula for Eddington luminosity (cf. for details pages 109-111, 408-409 of Kembhavi \& Narlikar 1999) one arrives at black hole masses of the order $10^{8} \mathrm{M}_{\odot}$ on the basis of perfect efficiency of energy conversion. An efficiency of $\leq 0.01$ would drive the mass up a hundred fold at least, i.e. to $10^{10} \mathrm{M}_{\odot}$ or greater. So far there is no direct evidence in any galaxy for such large dark masses. The largest masses which have been reliably estimated are about $10^{9} \mathrm{M}_{\odot}$.

In general it is necessary to explain where the bulk of the energy released which is not in the relativistic particle beams, is to be found. A possible explanation is that it is much of this energy which heats the diffuse gas in active galaxies giving rise to the extended X-ray emission in clusters and galaxies.

An even harder problem is to explain how the massive black holes in galaxies were formed in the first place. Were they formed before the galaxies or later? In the standard model both scenarios have been tried, but no satisfactory answer has been found.

In our model the energy comes with creation in the very strong gravitational fields very close to the central NBH, where the process can be much more efficient than can be expected in the tortuous chain envisaged in the classical gravitational picture. We shall discuss this in Section 7.

Would very massive galaxies result if the universe allows indefinitely large time for galaxy formation? Earlier ideas (Hoyle, 1953, Binney 1977, Rees and Ostriker 1977, Silk 1977) seemed to suggest so. In the present case two effects intervene to make massive galaxies rather rare. The first one is geometrical. Because of steady long-term expansion, the distance between two galaxies formed, say, $n$ cycles ago, would have increased by a factor $\sim \exp n Q / P$, and their density decreased by the factor $\sim \exp -3 n Q / P$. For $n \gg 1$, we expect the chance of finding such galaxies very small.

The second reason working against the growth of mass in a region comes from the negative energy and pressure of the $C$-field. As the mass grows through creation, the $C$-field also mounts and its repulsive effect ultimately causes enough instability for the mass to break up. Thus the large mass grows smaller by ejecting its broken parts.

What is ejected in an MCE? Are the ejecta more in the form of particles or radiation or coherent objects? All three are produced. For a discussion of the mechanism leading to ejection of coherent objects, see Hoyle, et al. (2000), Chapter 18. 


\subsection{Quasi-Stellar Objects}

In the early 1960s QSOs were discovered (Matthews and Sandage 1963; Schmidt 1963; cf. Burbidge and Burbidge 1967 for an extensive discussion) as star-like objects with large redshifts. Very early on, continuity arguments led to the general conclusion that they are very similar to the classical Seyfert glaxies, i.e. they are the nuclei of galaxies at much greater distances. However, also quite early in the investigations, it became clear that a good case could also be made for supposing that they are more likely to be compact objects ejected from comparatively local, low redshift active galaxies (Hoyle and Burbidge 1966).

After more than thirty years of controversy this issue has not yet been settled, but a very strong case for this latter hypothesis based on the observations of the clustering of many QSOs about active galaxies has been made. (Burbidge et al. 1971; Arp 1987; Burbidge 1996).

If this is accepted, it provides direct evidence that in the creation process active galaxies are able to eject compact sources with large intrinsic redshifts. What was not predicted was the existence of intrinsic redshifts. They present us with an unsolved problem, but one which must be closely connected to the creation process. A remarkable aspect of this problem is that the intrinsic redshifts show very clear peaks in their distribution with the first peak at $z=0.061$ and with a periodicity of the form $\triangle \log (1+z)=0.089$ (cf. Karlsson 1971, Burbidge and Napier 2001). The periodicity is in the intrinsic redshift component $\left(z_{i}\right)$, and in order to single out that component, either the cosmological redshift component $z_{c}$ must be very small i.e., the sources must be very close to us, or it must be known and corrected for by using the relation $\left(1+z_{o b s}\right)=\left(1+z_{c}\right)\left(1+z_{i}\right)$. Thus a recent claim that the periodicity is not confirmed (Hawkins et al., 2003) has been shown to be in error (Napier and Burbidge, 2003).

It is admitted that the evidence from gravitational lensing provides an overall consistent picture for the standard cosmological hypothesis. The evidence on quasars of larger redshift being lensed by a galaxy of lower redshift, together with the time delay in the radiation found in the two lensed images can be explained by this hypothesis. This type of evidence needs to be looked at afresh if the claim is made that quasars are much closer than their redshift-distances. In such cases, the lensing models can be 'scaled' down but the time-delay will have to be checked for lower values. To our knowledge no 
such exercise has been carried out to date. We hope to examine this issue in a later paper.

\subsection{Gamma Ray Bursts}

One of the most remarkable phenomena discovered in recent years relate to very short lived ( $\lesssim$ minutes) bursts of high energy photons ( $\gamma$-ray and x-ray) which can apparently occur anywhere in the sky, and which sometimes can be identified with a very faint optical and/or radio source, an afterglow, which may fade with time. Sometimes a very faint object remains. The first optical observation in which a redshift could be measured led to the conclusion that those sources are extragalactic. Using the redshifts as distance indicators this has led to the conclusion that the energies emitted lie in the range $10^{50}-10^{54}$ ergs, with most of them $\gtrsim 10^{53}$ ergs, if the explosions take place isotropically. If energies involving single stars are invoked the energies can be reduced if beaming is present. The most recent observations have suggested that the events are due to forms of supernovae which are beamed. In the usual interpretation it is assumed that the redshifts which have been measured for the gamma ray bursts are cosmological (cf Bloom et al. 2003). However in a recent study using all (more than 30) gamma-ray bursts (GRBs) with measured redshifts it was shown that the redshift distribution strongly suggests that they are closely related to QSOs with the same intrinsic redshift peaks (Burbidge 2003, 2004). Also an analysis of the positions of all of the GRBs for which we have positions (about 150) shows that a number of them are very near to already identified QSOs (Burbidge 2003). All of this suggests that the GRBs are due to explosions of objects (perhaps in QSOs) which have themselves been ejected following a creation process from active galax-

ies. In general they have slightly greater cosmological redshifts and thus are further away $(\leq 500 \mathrm{Mpc})$ than the galaxies from which most of bright QSOs are ejected. While we do not claim that this hypothesis is generally accepted, Bloom (2003) has shown that there are peculiarities in the redshift distribution interpreted in the conventional way. More observations may clarify this situation. 


\section{Dynamics and Spectrum of Radiation from a MCE}

A discussion of how a minicreation event arises was given in section 3. Thus we took the modified problem of a collapsing dust ball in the presence of the $C$-field as a toy-model of how a realistic massive object would behave. In the classic Oppenheimer-Snyder case the dust ball collapses to become a black hole, eventually ending in spacetime singularity. In the modified problem, as we saw in section 3, the dust ball need not become a black hole. It certainly does not attain singularity, but bounces at a finite radius. We saw that after bounce its outward speed rapidly rises before it ultimately slows down to a halt. In the phase of rapid expansion it resembles the classical white hole which is the reverse of the classical collapse without the $C$-field. The white hole solution can be used to approximate the behaviour of an MCE as seen by an external observer, because the former can be handled exactly in analytic way. In essence we use the notation of section 3 with slight modification.

We begin with a discussion of a white hole as considered by Narlikar et al (1974) within the framework of standard general relativity. Consider a massive object emerging from a spacetime singularity in the form of an explosion. To simplify matters Narlikar, Apparao and Dadhich (op. cit.) considered the object as a homogeneous dust ball, for which one can use comoving coordinates. As described in section 3, the line element within the object is given by

$$
\mathrm{d} s^{2}=\mathrm{d} t^{2}-a^{2}(t)\left[\frac{\mathrm{d} r^{2}}{\left(1-\alpha r^{2}\right)}+r^{2}\left(\mathrm{~d} \theta^{2}+\sin ^{2} \theta \mathrm{d} \phi^{2}\right)\right]
$$

where $c=$ speed of light is taken as unity, $a(t)$ is the expansion factor and $\alpha$ is a parameter related to the mass $M$ and the comoving radius $r_{b}$ of the object by

$$
2 G M=\alpha r_{b}^{3}
$$

The similarity of equation (48) to the Robertson-Walker line element of cosmology is well known. Also, if we change $t$ to $-t$, equation (48) represents

a freely collapsing ball of dust. The parameter $\alpha$ is related to the dust density $\rho_{0}$ at $a=1$, by the relation 


$$
\alpha=\frac{8 \pi G \rho_{0}}{3} .
$$

The formulae (48) - (50) are the same as (5), (10) and (6) of section 3. However, in $\S 3$ we were discussing the contracting phase, while here we are interested in the expanding mode. For convenience therefore, we will measure $t$ from the instant of explosion so that $a(0)=0$. For $t>0, a(t)$ satisfies the equation

$$
\dot{a}^{2}=\frac{\alpha(1-a)}{a},
$$

so that it attains its maximum value $a=1$ at

$$
t=t_{0}=\frac{\pi}{(2 \sqrt{ } \alpha)}
$$

We will investigate light emission from the white hole in the interval $0<t<$ $t_{0}$. The equation (51) can be solved in a parametric form by defining

$$
a=\sin ^{2} \xi, \quad 0 \leq \xi \leq \pi / 2 .
$$

The $\xi$ is related to the comoving time coordinate $t$ by

$$
t=\frac{2 t_{0}}{\pi}(\xi-\sin \xi \cos \xi)
$$

The white hole bursts out of the Schwarzschild radius at $t=t_{c}, \xi=\xi_{c}$, where

$$
\sin \xi_{c}=\left(\alpha r_{b}^{2}\right)^{1 / 2} .
$$

The space exterior to the white hole is described by the Schwarzschild line element

$$
\begin{array}{r}
\mathrm{d} s^{2}=[1-(2 G M / R)] \mathrm{d} T^{2}-\frac{\mathrm{d} R^{2}}{1-(2 G M / R)} \\
-R^{2}\left(\mathrm{~d} \theta^{2}+\sin ^{2} \theta \mathrm{d} \phi^{2}\right) .
\end{array}
$$

A typical Schwarzschild observer has $R=$ constant, $\theta=$ constant, $\phi=$ constant. We wish to calculate the spectrum of radiation from the white hole as seen by a Schwarzschild observer with $R=R_{1} \gg 2 G M$. To simplify matters 
further, we will take the luminosity spectrum of the white hole as $L \delta\left(\nu-\nu_{0}\right)$, where $L=$ constant.

Suppose two successive light signals are sent out from the surface at comoving instants $t$ and $t+d t$ and are received by the observer at $R_{1}$ at instants $T$ and $T+\mathrm{d} T$ measured in the Schwarzschild coordinates. Then a straightforward calculation shows that

$$
\frac{\mathrm{d} T}{\mathrm{~d} t}=\frac{\sin \xi}{\sin \left(\xi+\xi_{c}\right)}
$$

So an electromagnetic wave of frequency $\nu_{0}$ emitted from the surface appears to the receiver to have the frequency

$$
\nu=\nu_{0}\left[\frac{\sin \left(\xi+\xi_{c}\right)}{\sin \xi}\right] .
$$

A result of this type is suitable for working out the spectrum of the radiation as seen by the Schwarzschild observer. Under our assumption $L / h \nu_{0}$ photons of frequency $\nu_{0}$ are being emitted per unit $t$ - time from the surface. The number emitted in the interval $[t, t+\mathrm{d} t]$ is therefore $L \mathrm{~d} t / h \nu_{0}$. The same number must be received in the interval $[T, T+\mathrm{d} T]$, but with frequencies in the range $(\nu, \nu+\mathrm{d} \nu)$ where $\mathrm{d} \nu$ is related to $\mathrm{d} t$ through equations (54) and (58). A simple calculation gives

$$
\mathrm{d} t=\frac{\left(4 t_{0} \nu_{0}^{3} \sin ^{3} \xi_{c} \mathrm{~d} \nu\right)}{\pi\left(\nu^{2}+\nu_{0}^{2}-2 \nu \nu_{0} \cos \xi_{c}\right)^{2}} .
$$

Writing $E=h \nu, E_{0}=h \nu_{0}$, the number of photons in the range $[E, E-$ $\mathrm{d} E]$ received from the white hole per unit area at $R=R_{1}$ is given by

$$
N(E) \mathrm{d} E=\frac{L t_{0}}{\pi^{2} R_{1}^{2}} \times \frac{E_{0}^{2} \sin ^{3} \xi_{c} \mathrm{~d} E}{\left(E^{2}+E_{0}^{2}-2 E E_{0} \cos \xi_{c}\right)^{2}} .
$$

For $E \gg E_{0}$

$$
N(E) \mathrm{d} E \cong L t_{0} E_{0}^{2} \times \frac{\sin ^{3} \xi_{c}}{\pi^{2} R_{1}^{2}} \frac{\mathrm{d} E}{E^{4}} .
$$

The energy spectrum $I(E)$ is given by

$$
I(E)=E N(E) \propto E^{-3} .
$$


This is the spectrum at the high energy end under the simplifying assumptions made here. More general (and perhaps more realistic) assumptions can lead to different types of spectra which can also be worked out. Following Narlikar et al (1974) possible fields in high energy astrophysics where MCEs might find applications are as follows.

(i) The hard electromagnetic radiation from the MCEs situated at the centres of, say Seyfert galaxies, can be a source of background X and gamma radiation. The energy spectrum (60) seems, at first sight to be too steep compared to the observed spectrum $\propto E^{-1.2}$. But absorption effects in the gas present in the nuclei surrounding the MCE tend to flatten the spectrum given by equation (60). Detailed calculation with available data shows that these absorption effects can in fact flatten the $E^{-3}$ spectrum to $\sim E^{-1}$ form in the range $0.2 \mathrm{keV}$ to $1 \mathrm{keV}$. At lower energies, the ultraviolet radiation seems to be of the right order of magnitude to account for the infrared emission of $\sim 10^{45} \mathrm{erg} \mathrm{s}^{-1}$ through the dust grain heating mechanism.

(ii) The transient nature of X-ray and gamma-ray bursts suggests an MCE origin. The shape of the spectrum at the emitting end is likely to be more complicated than the very simple form assumed in the above example. In general, however, the spectrum should soften with time.

(iii) Although Narlikar et al. (1974) had worked out the spectrum of photons, it is not difficult to see that similar conclusions will apply to particles of nonzero rest mass provided they have very high energy, with the relativistic $\gamma-$ factor $\gg 1$. It is possible therefore to think of MCEs in the Galaxy on the scale of supernovae, yielding high energy cosmic rays right up to the highest energy observed.

This picture of a white hole gives a quantitative but approximate description of radiation coming out of an MCE, which is without a singular origin and without an event horizon to emerge out of. Ideally we should have used the modified $C$-field solution described in section 3 to calculate the exact result. This, however has proved to be an intractable problem analytically as an explicit exterior solution is not known.

The collapse problem with an earlier version of the $C$-field was discussed by Hoyle and Narlikar (1964) in which a proof was given that an exterior solution matching the homogeneous dust ball oscillation exists. However an explicit solution could not be given. The same difficulty exists with this solution also and further work, possibly using numerical general relativity, may be required. We mention in passing that a similar matching problem 
exists in inflationary models where a Friedmann bubble emerges within an external de Sitter type universe.

The above type of expansion has one signature. Its explosive nature will generate strong blueshifts, thus making the radiation of high frequency, which softens to that at lower frequencies as the expansion slows down. This model therefore has the general features associated with gamma ray bursts and transient X-ray bursters.

A further generalization of this idea at a qualitative level corresponds to the introduction of spin so as to correspond to the Kerr solution in classical general relativity. If we consider an MCE to have axial symmetry because of spin, the tendency to go round the axis is strong in a region close to the 'equator' and not so strong away from it. In classical general relativity the ergosphere identifies such a region: it shrinks to zero at the poles. At the poles therefore we expect that the ejection outwards will be preferentially directed along the axis and so we may see jets issuing in opposite directions.

In the very first paper on the QSSC, Hoyle, et al. (1993) had pointed to the similarity between an MCE and the standard early universe. In particular they had shown that the creation of matter in the form of Planck particles leads to their subsequent decay into baryons together with release of very high energy. These 'Planck fireballs' have a density temperature relationship of the form $\rho \alpha T^{3}$ which permits the synthesis of light nuclei just as in the classical big bang model. However, these authors drew attention to the circumstance that the relevant $(\rho, T)$ domain for this purpose in the QSSC is very different from the $(\rho, T)$ domain in the primordial nucleosynthesis of standard cosmology.

\section{Concluding Remarks}

The oscillating universe in the QSSC, together with a long-term expansion, driven by a population of mini-creation events provides the missing dynamical connection between cosmology and the 'local' explosive phenomena. The QSSC additionally fulfills the roles normally expected of a cosmological theory, namely (i) it provides an explanation of the cosmic microwave background with temperature, spectrum and inhomogeneities related to astrophysical processes (Narlikar et al. 2003), (ii) it offers a purely stellar-based interpretation of all observed nuclei (including light ones)(Burbidge et al. 
1957; Burbidge and Hoyle 1998), (iii) it generates baryonic dark matter as part of stellar evolution (Hoyle et al. 1994), (iv) it accounts for the extra dimming of distant supernovae without having recourse to dark energy (Narlikar, Vishwakarma and Burbidge 2002; Vishwakarma and Narlikar 2005), and it also suggests a possible role of MCEs in the overall scenario of structure formation (Nayeri et al. 1999).

The last mentioned work shows that preferential creation of new matter near existing concentrations of mass can lead to growth of clustering. A toy model based on million-body simulations demonstrates this effect and leads to clustering with a 2-point correlation function with index close to -1.8. Because of repulsive effect of the $C$-field, it is felt that this process may be more important than gravitational clustering. However, we need to demonstrate this through simulations like those in our toy model, together with gravitational clustering.

There are two challenges that still remain, namely understanding the origin of anomalous redshifts and the observed periodicities in the redshifts. Given the QSSC framework, one needs to find a scenario in which the hitherto classical interpretation of redshifts is enriched further with inputs of quantum theory. These are huge problems which we continue to wrestle with.

\section{Acknowledgements}

One of us, (JVN) thanks College de France, Paris for hospitality when this work was in process. RGV is grateful to IUCAA for hospitality which facilitated this collaboration. 


\section{References}

Ambartsumian, V.A. 1965, Structure and Evolution of Galaxies, Proc. 13th Solvay Conf. on Physics, University of Brussels, (New York, Wiley Interscience), 24

Arp, H.C. 1987, Quasars, Redshifts and Controversies (Interstellar Media, Berkeley, California)

Bagla, J.S., Padmanabhan, T. and Narlikar, J.V. 1996, Comm. Astrophys., 18, 289

Barrow, J., Kimberly, D. and Magueijo, J. 2004, Class. Quant. Grav., 21, 4289

Binney, J. 1977, Ap.J., 215, 483

Blanchard, A., Souspis, B., Rowan-Robinson, M. and Sarkar, S. 2003, A\&A, 412, 35

Bloom, J.S. 2003, A.J., 125, 2865

Bloom, J.S., Kulkarni, S.R. and Djorgovsky, S.G. 2001, A.J., 123, 1111

Bondi, H. and Gold, T. 1948, MNRAS, 108, 252

Burbidge, E.M., Burbidge, G.R., Fowler, W.A. and Hoyle, F. 1957, Rev. Mod. Phys., 29, 547

Burbidge, E.M., Burbidge, G., Solomon, P. and Strittmatter, P.A. 1971, Ap.J., 170, 223

Burbidge, G. 1975, Ap.J., 106, L7

Burbidge, G. 1996, A\&A, 309, 9 
Burbidge, G. 2003, Ap.J., 585, 112

Burbidge, G. 2004, "The Restless High Energy Universe", Conf. Proc. Nuclear Physics B., 305, 132

Burbidge, G. and Burbidge, E.M. 1965, The Structure and Evolution of Galaxies, Proc. of 13th Solvay Conference on Physics, University of Brussels, (New York, Wiley Interscience), 137

Burbidge, G. and Burbidge, E.M. 1967, Quasi-Stellar Objects, (San Francisco, W.H. Freeman)

Burbidge, G. and Hoyle, F. 1998, ApJ., 509, L1

Burbidge, G. and Napier, W. M. 2001, A.J., 121, 21

Burbidge, G., Burbidge, E.M., and Sandage, A. 1963, Rev. Mod. Phys., 35, 947

Carlstrom, J., Holder, G. and Reese, E. 2002, A.R.A.A., 40, 643

Carroll, S.M. and Press, W.H. 1992, A.R.A.A., 30, 499

Churasov, E., Sunyaev, R., Forman, W. and Bohringer, H. 2002, MNRAS, 332, 729

Datt, B. 1938, Z. Phys., 108, 314

Fabian, A.C. 1994, A.R.A.A., 32, 277

Fort, B. and Mellier, Y. 1994, AøA Rev., 4, 239

Gliner, E.B. 1970, Soviet Physics-Doklady, 15, 559

Gunn, J.B. and Oke, J.B. 1975, Ap.J., 195, 255 
Hawking, S.W. and Ellis, G.F.R. 1973, The Large Scale Structure of Spacetime, Cambridge

Hawkins, E., Maddox, S.J. and Merrifield, M.R. 2002, MNRAS, 336, L13

Hickson, P. 1997, A.R.A.A., 35, 377

Hogarth, J.E. 1962, Proc. R. Soc., A267, 365

Hoyle, F. 1948, MNRAS, 108, 372

Hoyle, F.1953, Ap.J, 118, 513

Hoyle, F. and Burbidge, G. 1966, Ap.J., 144, 534

Hoyle, F. and Narlikar, J.V 1963, Proc. Roy. Soc., A277, 1

Hoyle, F. and Narlikar, J.V. 1964, Proc. Roy. Soc., A278, 465

Hoyle, F. and Narlikar, J.V. 1969, Ann. Phys. (N.Y.), 54, 207

Hoyle, F. and Narlikar, J.V. 1971, Ann. Phys. (N.Y.), 62, 44

Hoyle, F. and Narlikar, J.V. 1995, Rev. Mod. Phys., 61, 113

Hoyle, F., Burbidge, G. and Narlikar, J.V. 1993, Ap.J, 410, 437

Hoyle, F., Burbidge, G. and Narlikar, J.V. 1994, MNRAS, 267, 1007

Hoyle, F., Burbidge, G. and Narlikar, J.V. 1995, Proc. Roy. Soc., A448, 191

Hoyle, F., Burbidge, G. and Narlikar, J.V. 2000, A Different Approach to Cosmology, (Cambridge, Cambridge University Press).

Hoyle, F., Fowler, W.A., Burbidge, E.M. and Burbidge, G. 1964, Ap.J, 139, 909 
Hoyle, F. and Sandage, A. 1956, P.A.S.P., 68, 301

Karlsson, K.G. 1971, A\&3A, 13, 333

Kembhavi, A.K. and Narlikar, J.V. 1999, Quasars and Active Galactic Nuclei, (Cambridge, Cambridge University Press).

Longair, M.S. 1987, IAU Symposium 124, "Observational Cosmology", (Editors, A. Hewitt, G. Burbidge, L.Z. Fang: D. Reidel, Dordrecht) p. 823

Matthews, T.A. and Sandage, A.R. 1963, Ap.J., 138, 30

McCrea, W.H. 1951, Proc.Roy.Soc., A206, 562

Meyers, A.D., Shanks, T., Outram, J.J., Srith, W.J. and Wolfendale, A.W. 2004, MNRAS, 347, L67

Napier, W. and Burbidge, G. 2003, MNRAS, 342, 601

Narlikar, J.V. 1973, Nature, 242, 35

Narlikar, J.V. and Padmanabhan, T. 1985, Phys. Rev. D32, 1928

Narlikar, J.V., Apparao, M.V.K. and Dadhich, N.K. 1974, Nature, 251, 590

Narlikar, J.V., Vishwakarma, R.G. and Burbidge, G. 2002, P.A.S.P., 114, 1092

Narlikar, J.V., Vishwakarma, R.G., Hajian, A., Souradeep, T., Burbidge, G. and Hoyle, F. 2003, Ap.J., 585, 1

Nayeri, A., Engineer, S., Narlikar, J.V. and Hoyle, F. 1999, Ap.J., 525, 10

Ostriker, J.P., Peebles, P.J.E. and Yahil, A. 1974, Ap.J., 193, L1

Page L., et al., 2003, Astrophys. J. Suppl. 148, 233 
Perlmutter, S. et al. 1999, Ap.J., 517, 565

Podariu, S., Souradeep, T., Gott III, J. R., Ratra, B. and Vogeley, M. S. 2001, Ap. J. S., 559, 9

Rees, M.J. 1984, A.R.A.A., 22, 471

Rees, M.J. and Ostriker, J.P. 1977, MNRAS, 179, 541

Riess, A. et al. 1998, A.J., 116, 1009

Rubano, C. and Seudellaro, P. 2004, astro-ph / 0410260

Sachs, R., Narlikar, J.V. and Hoyle, F. 1996, A\&A, 313, 703

Sami, M. and Toporensky, A. 2004, Mod. Phys. Lett. A, 19, 1509

Schmidt, M. 1963, Nature, 197, 1040

Silk, J. 1977, Ap.J, 211, 638

Singh, P., Sami, M. and Dadhich, N. 2003, Phys. Rev., D68, 023522

Spergel, D. et al. 2003, Ap.J.S., 148, 175

Spergel, D.N., et al., 2006, astro-ph/0603449

Steinhardt, P.J. and Turok, N. 2002, Science, 296, 1436

Toomre, A. and Toomre, J. 1972, Ap.J., 178, 623

Vishwakarma, R.G. and Narlikar, J.V. 2005, Int.J.Mod.Phys.D, 14, 2, 345

Wheeler, J.A. and Feynman, R.P. 1945, Rev. Mod. Phys., 17, 157

Wheeler, J.A. and Feynman, R.P. 1949, Rev. Mod. Phys., 21, 425 
Wickramasinghe, N.C. 2005, Current Issues in Cosmology, Proceedings of the Colloquium on 'Cosmology: Facts and Problems', Paris. (Cambridge, Cambridge University Press), 152 
Appendix : Field Theory Underlying the QSSC

Following Mach's principle, we begin with the hypothesis that inertia of any particle of matter owes its origin to the existence of all other particles of matter in the universe. If the particles are labelled $a, b, c, \ldots$ and the element of proper time of $a^{\text {th }}$ particle in Riemannian spacetime is denoted by $d s_{a}$, then we express the inertia of particle $a$ by the sum

$$
M_{a}(A)=\sum_{b \neq a} \int \lambda_{b} \tilde{G}(A, B) d s_{b}=\sum_{b \neq a} M^{(b)}(A) .
$$

where $A$ is a typical point on the world line of particle $a . \tilde{G}(A, B)$ is a scalar propagator communicating the inertial effect from $B$ to $A$. The coupling constant $\lambda_{b}$ denotes the intensity of the effect and without loss of generality may be set equal to unity. Likewise we may replace $M_{a}(A)$ by a scalar mass function $M(X)$ of a general spacetime point $X$, denoting the mass acquired by a particle at that point. As in Riemannian geometry we will denote by $R_{i k}$ the Ricci tensor and by $R$ the scalar curvature.

The individual contributors to $M(X)$ are the scalar functions $M^{(b)}(X)$, which are determined by the propagators $\tilde{G}(X, B)$. The simplest theory results from choice of a conformally invariant wave equation for $M^{(b)}(X)$,

$$
\square M^{(b)}(X)+\frac{1}{6} R M^{(b)}(X)+M^{(b)}(X)^{3}=\int \frac{\delta_{4}(X, B)}{\sqrt{-g(B)}} d s_{b} .
$$

The expression on the right hand side identifies the worldline of $b$ as the source. Why conformal invariance? In a theory of long range interactions influences travel along light cones and light cones are entities which are globally invariant under a conformal transformation. Thus a theory which picks out light cones for global communication is naturally expected to be conformally invariant. (A comparison may be made with special relativity. The local invariance of speed of light for all moving observers leads to the requirement of local Lorentz invariance of a physical theory.)

Although the above equation is non-linear, a simplification results in the smooth fluid approximation describing a universe containing a larger number of particles. Thus $M(X)=\sum_{b} M^{(b)}(X)$ satisfies an equation 


$$
\square M+\frac{1}{6} R M+\Lambda M^{3}=\sum_{b} \int \frac{\delta_{4}(X, B)}{\sqrt{-g(B)}} d^{4} s_{b} .
$$

What is $\Lambda$ ? Assuming that there are $N$ contributing particles in a cosmological horizon size sphere, we will get

$$
\Lambda \approx N^{-2}
$$

since adding $N$ equations of the kind (A2) leads to the cube term having a reduced coefficient by this factor, because of the absence of cross products $M^{(b)} M^{(c)}$ type $(b \neq c)$. Typically the observable mass in the universe is $\sim 10^{22} M_{\odot}$ within such a sphere, giving $N \sim 2 \times 10^{60}$ if the mass is typically that of a planck particle. We shall return to this aspect shortly. With this value for $N$, we have

$$
\Lambda \approx 2.5 \times 10^{-121}
$$

With these definitions we now introduce the action principle from which the field equations can be derived. In particle-particle interaction form it is simply

$$
\mathcal{A}=-\sum_{a} \int M_{a}(A) d s_{a}
$$

Expressed in terms of a scalar field function $M(X)$, it becomes

$$
\begin{aligned}
\mathcal{A} & =-\frac{1}{2} \int\left(M_{i} M^{i}-\frac{1}{6} R M^{2}\right) \sqrt{-g} d^{4} x+\frac{1}{4} \Lambda \int M^{4} \sqrt{-g} d^{4} x \\
& -\sum_{a} \int \frac{\delta_{4}(X, A)}{\sqrt{-g(A)}} M(X) d s_{a} .
\end{aligned}
$$


For example, the variation $M \rightarrow M+\delta M$ leads to the wave equation (A2). The variation of spacetime metric gives rise to gravitational equations. The variation of particle world lines gives rise to another scalar field, however, if we assume the worldlines to have finite beginnings. This is where creation of matter explicitly enters the picture. The characteristic mass of a typical particle that can be constructed in the theory using the available fundamental constants $c, G$ and $\hbar$ is the Planck mass

$$
m_{P}=\left(\frac{3 \hbar c}{4 \pi G}\right)^{1 / 2}
$$

We shall assume therefore that the typical basic particle created is the Planck particle with the above mass. We shall take $\hbar=1$ in what follows. Imagine now the worldline of such a particle beginning at a world-point $A_{0}$.

A typical Planck particle $a$ exists from $A_{0}$ to $A_{0}+\delta A_{0}$, in the neighborhood of which it decays into $n$ stable secondaries, $n \simeq 6.10^{18}$, denoted by $a_{1}, a_{2}, \ldots a_{n}$. Each such secondary contributes a mass field $m^{\left(a_{r}\right)}(X)$, say, which is the fundamental solution of the wave equation

$$
\square m^{\left(a_{r}\right)}+\frac{1}{6} R m^{\left(a_{r}\right)}+n^{2} m^{\left(a_{r}\right)^{3}}=\frac{1}{n} \int_{A_{0}+\delta A_{0}} \frac{\delta_{4}(X, A)}{\sqrt{-g(A)}} d a,
$$

while the brief existence of $a$ contributes $c^{(a)}(X)$, say, which satisfies

$$
\square c^{(a)}+\frac{1}{6} R c^{(a)}+c^{(a)^{3}}=\int_{A_{0}}^{A_{0}+\delta A_{0}} \frac{\delta_{4}(X, A)}{\sqrt{-g(A)}} d a,
$$

Summing $c^{(a)}$ with respect to $a, b, \ldots$ gives

$$
c(X)=\sum_{a} c^{(a)}(X)
$$

the contribution to the total mass $M(X)$ from the Planck particles during their brief existence, while 


$$
\sum_{a} \sum_{r=1}^{n} m^{\left(a_{r}\right)}(X)=m(X)
$$

gives the contribution of the stable secondary particles.

Although $c(X)$ makes a contribution to the total mass function

$$
M(X)=c(X)+m(X)
$$

that is generally small compared to $M(X)$, there is the difference that, whereas $m(X)$ is an essentially smooth field, $c(X)$ contains small exceedingly rapid fluctuations and so can contribute significantly to the derivatives of $c(X)$. The contribution to $c(X)$ from Planck particles $a$, for example, is largely contained between two light cones, one from $A_{0}$, the other from $A_{0}+\delta A_{0}$. Along a timelike line cutting these two cones the contribution to $c(X)$ rises from zero as the line crosses the light cone from $A_{0}$, attains some maximum value and then falls back effectively to zero as the line crosses the second light cone from $A_{0}+\delta A_{0}$. The time derivative of $c^{(a)}(X)$ therefore involves the reciprocal of the time difference between the two light cones. This reciprocal cancels the short duration of the source term on the right-hand side of (A10). The factor in question is of the order of the decay time $\tau$ of the Planck particles, $\sim 10^{-43}$ seconds. No matter how small $\tau$ may be, the reduction in the source strength of $c^{(a)}(X)$ is recovered in the derivatives of $c^{(a)}(X)$, which therefore cannot be omitted from the gravitational equations.

The derivatives of $c^{(a)}(X), c^{(b)}(X), \ldots$ can as well be negative as positive, so that in averaging many Planck particles, linear terms in the derivatives do disappear. It is therefore not hard to show that after such an averaging the gravitational equations become

$$
\begin{aligned}
& R_{i k}-\frac{1}{2} g_{i k} R- 3 \Lambda m^{2} g_{i k}=\frac{6}{m^{2}}\left[-T_{i k}+\frac{1}{6}\left(g_{i k} \square m^{2}-m_{; i k}^{2}\right)\right. \\
&\left.+\left(m_{i} m_{k}-\frac{1}{2} g_{i k} m_{l} m^{l}\right)+\frac{2}{3}\left(c_{i} c_{k}-\frac{1}{4} g_{i k} c_{l} c^{l}\right)\right] .
\end{aligned}
$$


Since the same wave equation is being used for $c(X)$ as for $m(X)$, the theory remains scale invariant. A scale change can therefore be introduced that reduces $M(X)=m(X)+c(X)$ to a constant, or one that reduces $m(X)$ to a constant. Only that which reduces $m(X)$ to a constant, viz

$$
\Omega=\frac{m(X)}{m_{P}}
$$

has the virtue of not introducing small very rapidly varying ripples into the metric tensor. Although small in amplitude such ripples produce nonnegligible contributions to the derivatives of the metric tensor, causing difficulties in the evaluation of the Riemann tensor, and so are better avoided. Simplifying with (A14) does not bring in this difficulty, which is why separating of the main smooth part of $M(X)$ now proves an advantage, with the gravitational equations simplifying to

$$
\begin{gathered}
8 \pi G=\frac{6}{m_{P}^{2}}, \quad m_{P} \text { a constant, } \\
R_{i k}-\frac{1}{2} g_{i k} R+\lambda g_{i k}=-8 \pi G\left[T_{i k}-\frac{2}{3}\left(c_{i} c_{k}-\frac{1}{4} g_{i k} c_{l} c^{l}\right)\right] .
\end{gathered}
$$

We define the cosmological constant $\lambda$ by

$$
\lambda=-3 \Lambda m_{P}^{2} \approx-2 \times 10^{56} \mathrm{~cm}^{-2}
$$

This value falls within the normally expected region of the magnitude of the cosmological constant. Note, however, that its sign is negative! This has been the consequence of the Machian origin of the cosmological constant through the non-linear equations (A2), (A3).

It has been on (A17) that the discussion of what is called the quasi-steady state cosmological model (QSSC) has been based. A connection with the $C$-field of the earlier steady state cosmology can also be given. Writing 


$$
C(X)=\tau c(X)
$$

where $\tau$ is the decay lifetime of the Planck particle, the action contributed by Planck particles $a, b, \ldots$,

$$
-\sum_{a} \int_{A_{0}}^{A_{0}+\delta A_{0}} c(A) d a
$$

can be approximated as

$$
-C\left(A_{0}\right)-C\left(B_{0}\right)-\ldots,
$$

which form corresponds to the $C$-field used in the steady state cosmology.

Thus the equations (A17) are replaced by

$$
R_{i k}-\frac{1}{2} g_{i k} R+\lambda g_{i k}=-8 \pi G\left[T_{i k}-f\left(C_{i} C_{k}-\frac{1}{4} g_{i k} C_{l} C^{l}\right)\right],
$$

with the earlier coupling constant $f$ defined as

$$
f=\frac{2}{3 \tau^{2}}
$$

[We remind the reader that we have taken the speed of light $c=1$.]

The question now arises of why astrophysical observation suggests that the creation of matter occurs in some places but not in others. For creation to occur at the points $A_{0}, B_{0}, \ldots$ it is necessary classically that the action should not vary with respect to small changes in the spacetime positions of these points, which was shown earlier to require

$$
C_{i}\left(A_{0}\right) C^{i}\left(A_{0}\right)=C_{i}\left(B_{0}\right) C^{i}\left(B_{0}\right)=\ldots=m_{P}^{2}
$$

More precisely, the field $c(X)$ is required to be equal to $m_{P}$ at $A_{0}, B_{0}, \ldots$, 


$$
c\left(A_{0}\right)=c\left(B_{0}\right)=\ldots=m_{P} .
$$

(For, equation (A19) tells us that connection between $c$ and $C$ is through the lifetime $\tau$ of Planck particle.)

As already remarked in the main text, this is in general not the case: in general the magnitude of $C^{i} C_{i}$ is much less that $m_{P}$. However, close to the event horizon of a massive compact body $C_{i}\left(A_{0}\right) C^{i}\left(A_{0}\right)$ is increased by a relativistic time dilatation factor, whereas $m_{P}^{2}$ stays fixed. Hence, near enough to an event horizon the required conservation conditions can be satisfied, which has the consequence that creation events occur only in compact regions, agreeing closely with the condensed regions of high excitation observed so widely in astrophysics. 